\title{
Asymmetric N-Heterocyclic Carbene Catalyzed Annulation of 2-Alkenylbenzothiazoles with $\alpha$-Chloro Aldehydes
}

\author{
Xiaoxiao Song \\ Qijian Ni \\ Chen Zhu \\ Gerhard Raabe \\ Dieter Enders*
}

Institute of Organic Chemistry, RWTH Aachen University, Landoltweg 1, 52074 Aachen, Germany

enders@rwth-aachen.de

Dedicated to Professor Martin Jansen on the occasion of his $70^{\text {th }}$ birthday

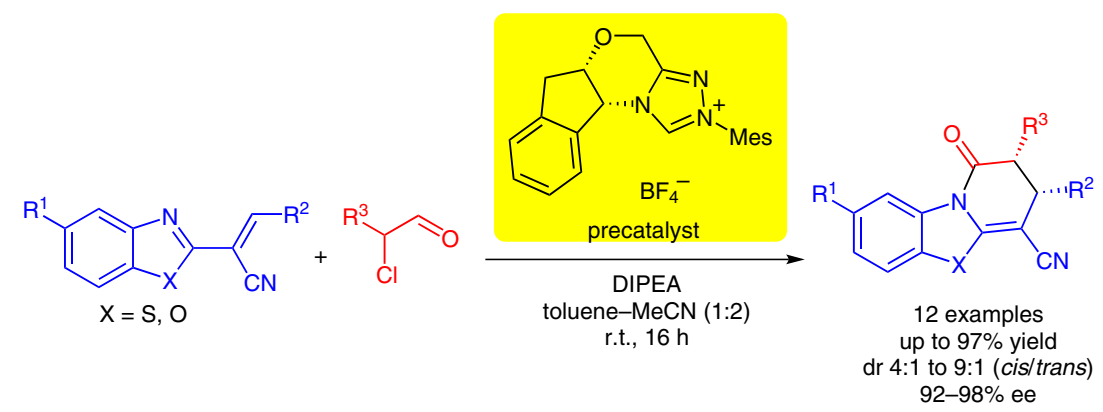

Received: 26.09 .2014

Accepted after revision: 30.09.2014

Published online: 06.11 .2014

DOI: 10.1055/s-0034-1379369; Art ID: ss-2014-z0591-op

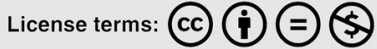

Abstract Diastereo- and enantioselective $\mathrm{N}$-heterocyclic carbene catalyzed 1-azadiene Diels-Alder reactions of (E)-2-styrylbenzothiazoles with $\alpha$-chloro aldehydes are reported. This annulation strategy provides an efficient access to medicinally important dihydrobenzothiazolopyridin-1-ones in good to excellent yields (44-97\%) with very good to excellent stereoselectivities (up to 9:1 dr, 98\% ee) and tolerates quite a range of substituents.

Key words N-heterocyclic carbenes, organocatalysis, asymmetric synthesis, aza-Diels-Alder reaction, annulation

The aza-Diels-Alder (aza-DA) reaction is one of the most powerful and elegant synthetic approaches to form $\mathrm{C}-\mathrm{C}$ and $\mathrm{C}-\mathrm{N}$ bonds, resulting in nitrogen-containing sixmembered heterocycles. ${ }^{1}$ Since Ghosez's pioneering work introducing the reaction of l-azadienes with electron-deficient dienophiles, ${ }^{2}$ followed by Danishefsky's report on the formation of piperidine rings from imines and electron-rich dienes, ${ }^{3}$ a great variety of aza-DA reactions under thermal conditions or catalyzed by Lewis acids ${ }^{4}$ have been reported. With the renaissance of organocatalysis, enantioselective aza-DA reactions catalyzed by amines, ${ }^{5}$ thioureas, ${ }^{6}$ or chiral phosphoric acids, ${ }^{7}$ were intensively investigated.

Over the past few decades, N-heterocyclic carbenes (NHCs), as an outstanding class of organocatalysts, have provided efficient access to various structural scaffolds through the Umpolung of aldehydes. ${ }^{8}$ In 2004, the groups of Glorius and Bode reported the generation of homoenolates via NHC catalysis. ${ }^{9}$ This provided an elegant way to activate the $\beta$-position of enals (conjugated Umpolung). Later, as an extended activation mode, azolium enolates were devel- oped and acted as reactive nucleophiles in various NHC-catalyzed reactions. ${ }^{10}$ Notably, the in situ generated azolium enolates paved the way to numerous important heterocyclic manifolds via $[2+2]^{11}$ and $[2+3]$ cycloadditions, ${ }^{12}$ as well as [2+4] inverse-electron-demand Diels-Alder reactions. ${ }^{13}$ In 2006, Bode and co-workers were the first to report a highly enantioselective aza-DA reaction of 1-azadienes with the enolate species generated in situ from enals. ${ }^{14}$ Afterwards, Ye and co-workers employed ketenes ${ }^{15}$ and $\alpha$ chloro aldehydes ${ }^{16}$ as substrates, which reacted with 1-azadienes in the presence of NHC catalysts. Then, Rovis and coworkers accessed the azolium enolate equivalents via oxidation of the Breslow intermediates, and constructed the corresponding lactams with azadienes. ${ }^{17}$ Recently, Chi and co-workers have reported an NHC-catalyzed reaction of $\alpha$ aryl esters with azadienes. ${ }^{18}$ However, these reported studies all utilized acyclic 1-azadienes as reaction partners (Scheme 1, eq 1). To the best of our knowledge, little attention has been given to aza-DA reactions of cyclic 1-azadienes with in situ generated azolium enolate species.

previous work:

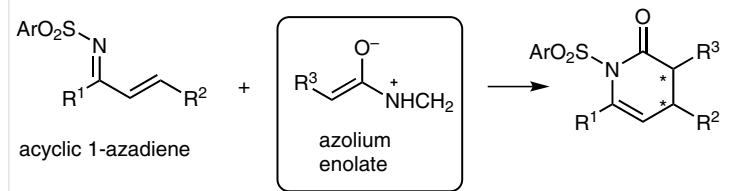

this work:

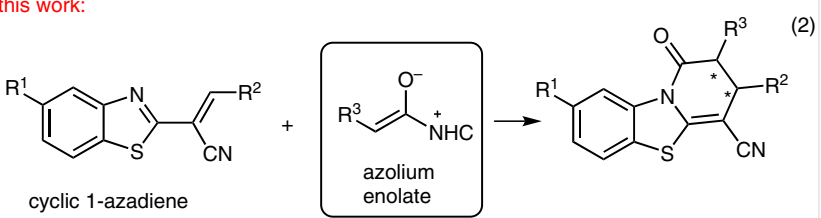

Scheme 1 NHC-catalyzed aza-Diels-Alder reactions 
Herein, we describe an enantioselective NHC-catalyzed cycloaddition of $(E)$-2-styrylbenzothiazoles with $\alpha$-chloro aldehydes (Scheme 1, eq 2). The resulting dihydro-1H-benzothiazolopyridine core is an important structural motif existing in various biologically active products and numerous pharmaceuticals, such as antitumor ${ }^{19}$ and antibacterial drugs. $^{20}$

Initially, we investigated the reaction of $(E)-2$-(benzothiazol-2-yl)-3-phenylacrylonitrile (1a) with 2-chloro-3phenylpropanal (2a) at room temperature in toluene in the presence of triethylamine and triazolium precatalyst $\mathbf{A}$. The reaction was finished within 16 hours and gave the aza-DA adduct 3a in a good cis/trans diastereoselectivity $(\mathrm{dr}=6: 1)$ and an excellent ee value (95\%) of the major cis-diastereomer, albeit in a low yield (29\%) (Table 1, entry 1). Other triazolium precatalysts B-D were also tested, but only traces (Table 1, entries 2 and 3 ) or even no product at all (Table 1 , entry 4) was obtained under the same conditions. In an attempt to increase the yield of the desired $\mathbf{3 a}$, we elevated the reaction temperature to $40{ }^{\circ} \mathrm{C}$, leading to no improvement (Table 1 , entry 5). Next, base screening showed that organic bases (such as TMEDA, DIPEA, or DABCO) gave better or similar yields as well as excellent stereoselectivities (Table 1, entries 6-8); however, inorganic bases (such as $\mathrm{NaOAc}$ or $\mathrm{K}_{2} \mathrm{CO}_{3}$ ) furnished only traces or no product at all (Table 1, entries 9 and 10). We then focused on optimizing a series of reaction solvents in the presence of precatalyst $\mathbf{A}$ and $N, N, N^{\prime}, N^{\prime}$-tetramethylethylenediamine at room temperature (Table 1, entries 11-14). Interestingly, an excellent yield (96\%) and enantioselectivity (96\% ee) was obtained in acetonitrile (Table 1, entry 14); however, the dr was inversed (cis/trans, 1:2.5) relative to the other solvents tested. In order to improve the $\mathrm{dr}$, we retested the organic bases in acetonitrile and obtained a better $\mathrm{dr}(3.5: 1)$ when $\mathrm{N}, \mathrm{N}$-diisopropylethylamine was used as base (Table 1, entry 16). Finally, we sought to adjust the solvent ratio of toluene and acetonitrile. Fortunately, the product was obtained with excellent yield and ee, as well as good $\mathrm{dr}(7: 1)$, in a mixed solvent of toluene-acetonitrile (1:2) (Table 1 , entry 20 ).

Under the optimized conditions, the scope of the reaction was evaluated on a $0.5-\mathrm{mmol}$ scale (Table 2 ). Initially, a variety of $(E)$-2-styrylbenzothiazoles 1 with different electronic properties were examined. Obviously, the electrondonating effect influenced the reaction reactivity and decreased yields of the desired products were obtained, while good $\mathrm{dr}$ and excellent ee values were observed $\left(\mathrm{R}^{2}=4-\mathrm{Tol}\right.$, 4- $\mathrm{MeOC}_{6} \mathrm{H}_{4}$; Table 2, 3b and $3 \mathbf{c}$ ). In the case of electronwithdrawing groups (such as 3-OMe, $4-\mathrm{Cl}, 3-\mathrm{Cl}$, or $4-\mathrm{F}$ ), the reactions were finished within 16 hours and gave the desired lactam adducts in good to excellent yields, good diastereoselectivities and excellent enantioselectivities (Table $2, \mathbf{3 d}-\mathbf{g})$. If $\mathrm{R}^{2}$ is a 2 -furyl group, a relatively low yield was obtained in 40 hours (Table 2, 3h). This may be due to the electron-donating effect of the furan ring. The variation of $\mathrm{R}^{1}$ on the benzothiazole motif $\mathbf{1}$ to a chloro substituent afforded the corresponding product $\mathbf{3 i}$ in $97 \%$ yield and excellent stereoselectivity ( $\mathrm{dr}=9: 1,98 \%$ ee $)$. Notably, this protocol could also be extended to (E)-2-(benzoxazol-2-yl)-3phenylacrylonitrile (1j), which produced product $\mathbf{3 j}$ in $97 \%$ yield, 4:1 dr, and 95\% ee. With regard to the $\alpha$-chloro aldehydes 2, aliphatic linear aldehydes are also tolerated, leading to the corresponding products $\mathbf{3 k}$ and $\mathbf{3 1}$ in moderate yields. The relative configuration of the major diastereomer of $\mathbf{3}$ was assigned by NOE measurements on 3d (see Supporting Information). It was clear that $\mathrm{H}^{\mathrm{a}}$ and $\mathrm{H}^{\mathrm{b}}$ are in a cis relationship, which is in accordance with the absolute configuration of compound 3d determined by X-ray crystal structure analysis (Figure 1). ${ }^{21}$
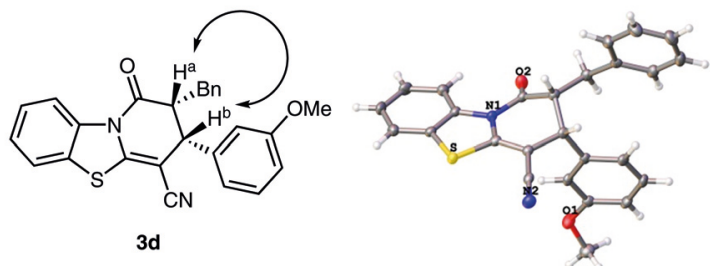

Figure 1 The relative (NOE) and absolute configuration [X-ray, Xabs = $0.049(18)]$ of $3 \mathbf{d}$

A possible mechanism explaining the cis configuration of the major diastereomer is shown in Scheme 2. Initially, nucleophilic addition of the free carbene $\mathbf{A}^{\prime}$ to the $\alpha$-chloro aldehyde $\mathbf{2}$ affords the adduct $\mathbf{I}$, followed by the removal of hydrogen chloride to form the enolate species II. Since the $R e$ face of the azolium enolate is blocked by the indane backbone of the NHC, the enolate undergoes an aza-DA reaction on the Si face with the (E)-2-styrylbenzothiazole 1, via the favored endo transition state endo-TS. ${ }^{13 i, 22}$ Subsequent $\alpha$-elimination leads to the final product 3 and liberates the NHC catalyst for further cycles. In a more asynchronous manner, the annulation can be seen as a Michael-lactamization sequence. The rather low ee values of the minor trans-diastereomer may be explained by the presence of an E/Z-azolium enolate mixture (see Supporting Information).

In summary, we have developed an efficient NHC-catalyzed asymmetric reverse-electron-demand 1-azadiene Diels-Alder reaction of $(E)$-2-styrylbenzothiazoles with $\alpha$ chloro aldehydes, resulting in the desired cis-dihydrobenzothiazolopyridin-1-ones in good to excellent yields, good diastereomeric ratios and excellent ee values. This annulation protocol tolerates quite a range of substrates, leading to medicinally interesting tricyclic dihydrobenzothiazolopyridines bearing a synthetically useful cyano group at the 4position. 
Table 1 Optimization of the Reaction Conditions ${ }^{\mathrm{a}}$<smiles>N#C/C(=C\c1ccccc1)c1nc2ccccc2s1</smiles>

1a<smiles>[PH3-][n+]1cn2c(n1)CO[C@@H]1Cc3ccccc3[C@@H]12</smiles>
A $R=$ Mes
B $R=\mathrm{C}_{6} \mathrm{~F}_{5}$

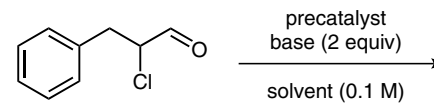

$2 a$<smiles>N#CC1=C2Sc3ccccc3N2C(=O)C(Br)[C@@H]1c1ccccc1</smiles>

$3 a$

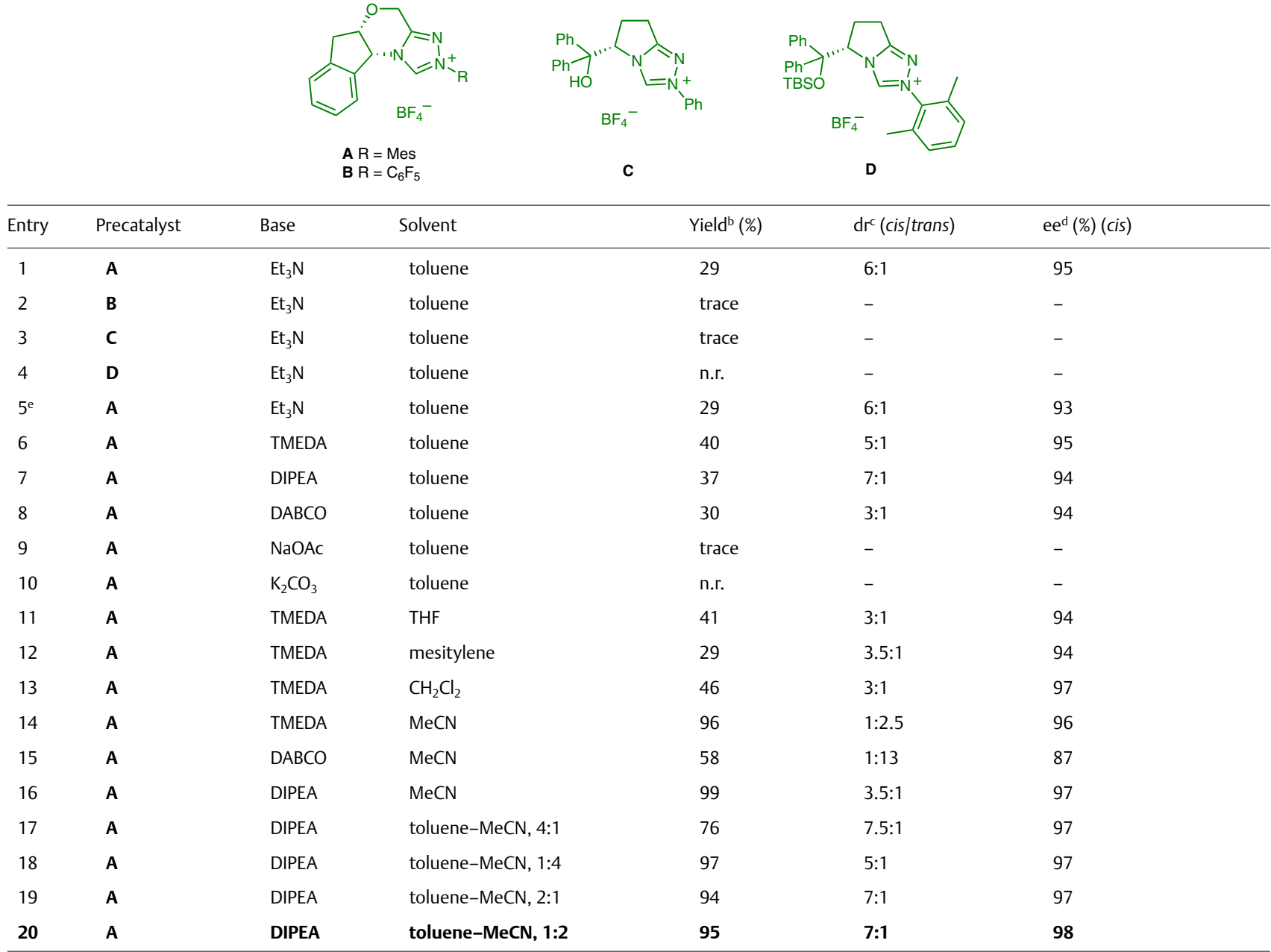

a Reaction conditions: 1a $(0.2 \mathrm{mmol}), 2 a(0.3 \mathrm{mmol})$, precatalyst $(0.02 \mathrm{mmol})$, base $(0.4 \mathrm{mmol})$, solvent ( $\mathrm{v} / \mathrm{v}, 2 \mathrm{~mL}$ in total), r.t., under argon, $16 \mathrm{~h}$.

b Yield of isolated product 3 a after column chromatography; n.r. = no reaction.

c Determined by ${ }^{1} \mathrm{H}$ NMR spectroscopy.

${ }^{d}$ Determined by HPLC on a chiral stationary phase.

e Performed at $40^{\circ} \mathrm{C}$

All reactions were carried out under argon. THF and toluene were distilled over Solvona ${ }^{\circledR} . \mathrm{CH}_{2} \mathrm{Cl}_{2}$ was distilled over $\mathrm{CaH}_{2}$. All other chemicals were used without further purification. Triazolium salts, chiral triazolium-derived carbene catalysts $\mathbf{A}-\mathbf{D}$ and the achiral triazoliumderived carbene catalyst 2-phenyl-6,7-dihydro-5H-pyrrolo[2,1c] $[1,2,4]$ triazol-2-ium tetrafluoroborate (which was used to synthesize the racemic products) were prepared according to known literature procedures. ${ }^{23}$ (E)-2-(Benzothiazol-2-yl)-3-phenylacrylonitriles were prepared from benzothiazol-2-ylacetonitriles. ${ }^{24} \alpha$-Chloro aldehydes were prepared from aldehydes. ${ }^{25}$ Chromatographic purification of the products was performed on Merck silica gel 60, particle size
0.040-0.063 mm (230-240 mesh, flash). Analytical TLC was performed on SIL G-25 UV254 plates (MACHEREY-NAGEL). The developed TLC plates were visualized with ultraviolet irradiation $(254 \mathrm{~nm})$. Optical rotation values were measured on a Perkin-Elmer 241 polarimeter. Microanalyses were performed with a Vario EL elemental analyzer. Mass spectra and high-resolution mass spectra were acquired on a Finnigan MAT $95(\mathrm{EI} / \mathrm{CI})$ or on a Thermo Fisher Scientific LTQ Orbitrap XL (ESI) mass spectrometer. IR spectra were recorded on a Perkin-Elmer FT-IR Spectrum 100 spectrometer using a Diamant/ KRS5 ATR attachment. Evaluation was undertaken using the supplementary software. The absorption bands are given in wavenumbers 
Table 2 Asymmetric Synthesis of Dihydrobenzothiazolopyridin-1-ones $3^{\text {a }}$

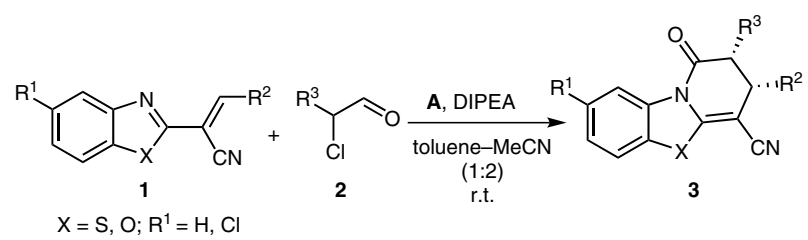

\begin{tabular}{|c|c|c|c|c|c|}
\hline 3 & $\mathrm{R}^{2}$ & $\mathrm{R}^{3}$ & Yield $(\%)$ & $\mathrm{dr}^{\mathrm{c}}$ & $e^{d}(\%)$ \\
\hline a & $\mathrm{Ph}$ & $\mathrm{Bn}$ & 93 & $7: 1$ & 96 \\
\hline b & 4-Tol & $\mathrm{Bn}$ & 74 & 9:1 & 96 \\
\hline$c^{e}$ & $4-\mathrm{MeOC}_{6} \mathrm{H}_{4}$ & Bn & 72 & $8: 1$ & 94 \\
\hline d & $3-\mathrm{MeOC}_{6} \mathrm{H}_{4}$ & $\mathrm{Bn}$ & 94 & $6: 1$ & 98 \\
\hline e & $4-\mathrm{ClC}_{6} \mathrm{H}_{4}$ & Bn & 84 & $6: 1$ & 97 \\
\hline$f$ & $3-\mathrm{ClC}_{6} \mathrm{H}_{4}$ & Bn & 97 & $4: 1$ & 98 \\
\hline g & 4- $\mathrm{FC}_{6} \mathrm{H}_{4}$ & $\mathrm{Bn}$ & 97 & $4: 1$ & 97 \\
\hline$h^{f}$ & 2-furyl & $\mathrm{Bn}$ & 68 & $6: 1$ & 92 \\
\hline $\mathbf{i}^{\mathrm{g}}$ & $\mathrm{Ph}$ & $\mathrm{Bn}$ & 97 & $9: 1$ & 98 \\
\hline $\mathrm{j}^{\mathrm{h}}$ & $\mathrm{Ph}$ & $\mathrm{Bn}$ & 97 & $4: 1$ & 95 \\
\hline k & $\mathrm{Ph}$ & $n-B u$ & 71 & $8: 1$ & 98 \\
\hline I & $\mathrm{Ph}$ & $n$-Hex & 44 & $5: 1$ & 96 \\
\hline
\end{tabular}

a Reaction conditions: $\mathbf{1}(0.5 \mathrm{mmol}), \mathbf{2}(0.75 \mathrm{mmol})$, precatalyst $\mathbf{A}(0.05$ mmol), DIPEA (1.0 mmol), toluene-MeCN (1:2, v/v; $5 \mathrm{~mL}$ in total), r.t., under argon, $16 \mathrm{~h} ; \mathrm{R}^{1}=\mathrm{H}, \mathrm{X}=\mathrm{S}$, unless otherwise noted.

${ }^{\mathrm{b}}$ Yield of isolated product $\mathbf{3}$ as a mixture of diastereomers after column chromatography.

${ }^{\mathrm{C}}$ Determined by ${ }^{1} \mathrm{H}$ NMR spectroscopy.

${ }^{\mathrm{D}}$ Determined by HPLC on a chiral stationary phase.

e Reaction time was $43 \mathrm{~h}$.

Reaction time was $40 \mathrm{~h}$

${ }^{g} \mathrm{R}^{1}=\mathrm{Cl}$; reaction time was $18 \mathrm{~h}$.

${ }^{\mathrm{h}} \mathrm{X}=\mathrm{O}$.

$\left(\mathrm{cm}^{-1}\right) .{ }^{1} \mathrm{H}$ and ${ }^{13} \mathrm{C}$ NMR spectra were recorded at ambient temperature on Varian VNMRS 600 and Inova 400 instruments. The chemical shifts are reported in ppm downfield from TMS and referenced to residual solvent peak resonances as internal standard. The order of citation of data in parentheses is a) multiplicity ( $\mathrm{s}=$ singlet, $\mathrm{d}=$ doublet, $\mathrm{dd}=$ doublet of doublet, $\mathrm{t}=$ triplet, $\mathrm{m}=$ multiplet), $\mathrm{b}$ ) coupling constants, and c) number of protons. Coupling constants $(J)$ are reported in hertz $(\mathrm{Hz})$. Analytical HPLC was performed on a Hewlett-Packard 1100 Series instrument using chiral stationary phases (Daicel AD, Daicel IA, Daicel IC).

\section{Compounds 3a-I; General Procedure}

To a dried and argon-filled Schlenk flask was added an (E)-2-styrylbenzothiazole 1 ( $0.5 \mathrm{mmol}, 1.0$ equiv), an $\alpha$-chloro aldehyde $\mathbf{2}(0.75$ mmol, 1.5 equiv), triazolium salt $\mathbf{A}(0.05 \mathrm{mmol}, 10 \mathrm{~mol} \%)$, and DIPEA $(1.0 \mathrm{mmol}, 2.0$ equiv) in a mixed solvent of toluene-MeCN $(1: 2, \mathrm{v} / \mathrm{v} ; 5$ $\mathrm{mL}$ ). The mixture was stirred at r.t. and monitored by TLC until completion of the reaction. After removal of the solvent under reduced pressure, the residue was purified by flash chromatography on silica gel (pentane- $\mathrm{Et}_{2} \mathrm{O}, 10: 1$ ) to afford the product $\mathbf{3 a - 1}$ as a colorless or light yellow solid.

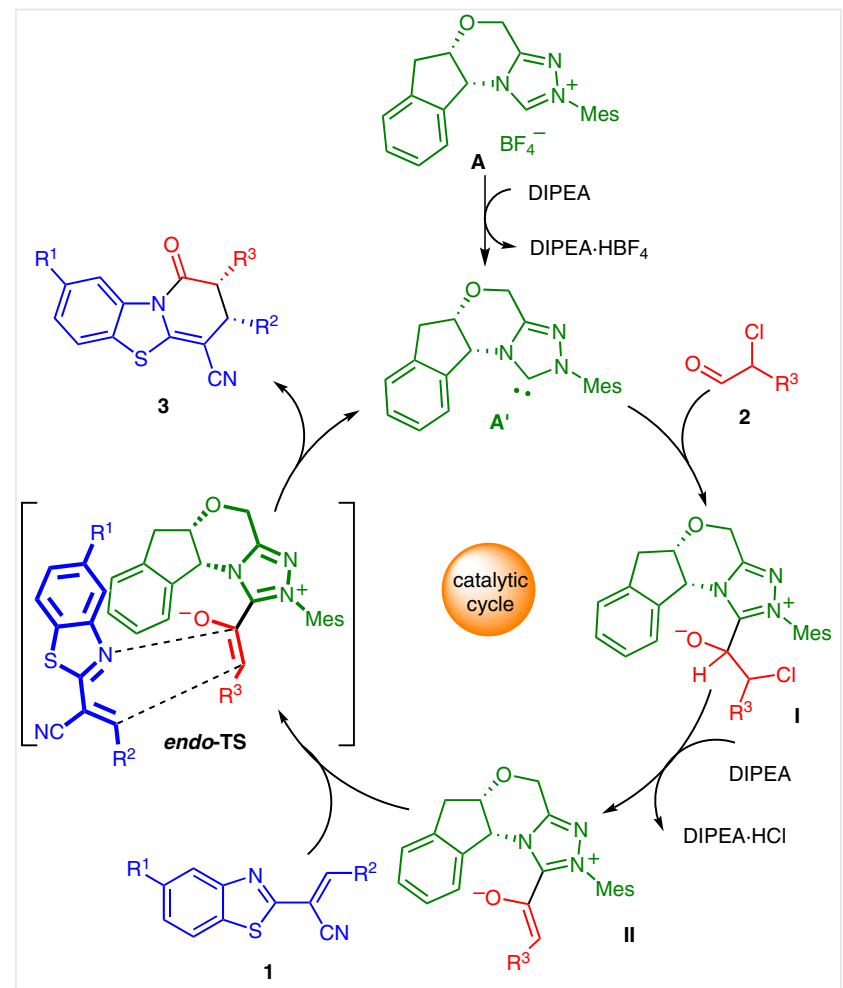

Scheme 2 Proposed reaction mechanism

(2R,3R)-2-Benzyl-1-oxo-3-phenyl-2,3-dihydro-1H-benzo[4,5]thiazolo[3,2-a]pyridine-4-carbonitrile (3a)

The ee (96\%) was measured by HPLC using a chiral stationary phase [Daicel IC; $n$-heptane-EtOH, 9:1, $0.5 \mathrm{~mL} / \mathrm{min} ; t_{\mathrm{R}}=11.01 \mathrm{~min}$ (minor), 13.47 min (major)]

Yield: $183.2 \mathrm{mg}$ (93\%); colorless solid; $\mathrm{mp} 140-142{ }^{\circ} \mathrm{C}$.

$[\alpha]_{\mathrm{D}}^{21}-501.5\left(\right.$ c $\left.1.0, \mathrm{CHCl}_{3}\right)$.

IR (ATR): 3446, 3026, 2938, 2321, 2196, 2103, 1948, 1719, 1605, 1493 , 1455, 1364, 1314, 1223, 1135, 1065, 1027, 983, 919, 849, 748, 697 $\mathrm{cm}^{-1}$.

${ }^{1} \mathrm{H}$ NMR $\left(600 \mathrm{MHz}, \mathrm{CDCl}_{3}\right): \delta=8.44(\mathrm{~d}, J=8.4 \mathrm{~Hz}, 1 \mathrm{H}), 7.38-7.24(\mathrm{~m}, 9$ H), $7.07(\mathrm{~d}, J=7.2 \mathrm{~Hz}, 4 \mathrm{H}), 3.66-3.63(\mathrm{~m}, 1 \mathrm{H}), 3.55-3.52(\mathrm{~m}, 1 \mathrm{H})$, $3.46\left(\mathrm{dd}, J_{1}=14.4 \mathrm{~Hz}, J_{2}=4.2 \mathrm{~Hz}, 1 \mathrm{H}\right), 2.49\left(\mathrm{dd}, J_{1}=14.4 \mathrm{~Hz}, J_{2}=9.6\right.$ $\mathrm{Hz}, 1 \mathrm{H})$.

${ }^{13} \mathrm{C}$ NMR $\left(150 \mathrm{MHz}, \mathrm{CDCl}_{3}\right): \delta=169.3,152.3,138.1,137.5,136.5$, 129.3 (2 C), 128.9 (2 C), 128.7 (2 C), 128.5, 127.7 (2 C), 127.1, 126.8, $126.0,123.8,121.9,117.9,117.3,83.5,48.0,43.1,31.5$

MS (ESI): $m / z=395.1215[\mathrm{M}+\mathrm{H}]^{+}$.

HRMS (ESI): $m / z$ [M+Na] $]^{+}$calcd for $\mathrm{C}_{25} \mathrm{H}_{18} \mathrm{~N}_{2} \mathrm{OS}: 417.1032$; found: 417.1030.

(2R,3R)-2-Benzyl-1-oxo-3-(p-tolyl)-2,3-dihydro-1H-benzo[4,5]thiazolo[3,2-a]pyridine-4-carbonitrile (3b)

The ee $(96 \%)$ was measured by HPLC using a chiral stationary phase [Daicel IC; $n$-heptane- $i$-PrOH, 97:3, $1.0 \mathrm{~mL} / \mathrm{min} ; t_{\mathrm{R}}=16.05 \mathrm{~min}(\mathrm{mi}-$ nor), $27.22 \mathrm{~min}$ (major)].

Yield: $151.8 \mathrm{mg}$ (74\%); colorless solid; mp $133-135^{\circ} \mathrm{C}$. 
$[\alpha]_{\mathrm{D}}^{21}-509.4\left(c 1.0, \mathrm{CHCl}_{3}\right)$.

IR (ATR): 3441, 3024, 2928, 2324, 2196, 2100, 1978, 1718, 1606, 1508, $1456,1364,1313,1223,1134,1029,982,934,813,748,702 \mathrm{~cm}^{-1}$.

${ }^{1} \mathrm{H}$ NMR $\left(600 \mathrm{MHz}, \mathrm{CDCl}_{3}\right): \delta=8.43(\mathrm{~d}, J=8.4 \mathrm{~Hz}, 1 \mathrm{H}), 7.38-7.22(\mathrm{~m}, 6$ H), 7.13 (d, $J=7.8 \mathrm{~Hz}, 2 \mathrm{H}), 7.07$ (d, $J=7.2 \mathrm{~Hz}, 2 \mathrm{H}), 6.95$ (d, $J=8.4 \mathrm{~Hz}$, $2 \mathrm{H}), 3.60(\mathrm{~d}, J=6.6 \mathrm{~Hz}, 1 \mathrm{H}), 3.52-3.48(\mathrm{~m}, 1 \mathrm{H}), 3.44\left(\mathrm{dd}, J_{1}=14.4 \mathrm{~Hz}\right.$, $\left.J_{2}=4.8 \mathrm{~Hz}, 1 \mathrm{H}\right), 2.49\left(\mathrm{dd}, J_{1}=14.4 \mathrm{~Hz}, J_{2}=9.0 \mathrm{~Hz}, 1 \mathrm{H}\right), 2.34(\mathrm{~s}, 3 \mathrm{H})$.

${ }^{13} \mathrm{C}$ NMR $\left(150 \mathrm{MHz}, \mathrm{CDCl}_{3}\right): \delta=169.4,152.0,138.2,138.2,137.5$, 133.4, 129.9 (2 C), 128.9 (2 C), 128.7 (2 C), 127.5 (2 C), 127.1, 126.8, 125.9, 123.8, 121.8, 117.9, 117.3, 83.7, 48.1, 42.7, 31.5, 21.1.

MS (ESI): $m / z=409.1373[\mathrm{M}+\mathrm{H}]^{+}$.

HRMS (ESI): $m / z$ [M $+\mathrm{Na}]^{+}$calcd for $\mathrm{C}_{26} \mathrm{H}_{20} \mathrm{~N}_{2} \mathrm{OS}$ : 431.1189; found: 431.1188 .

\section{(2R,3R)-2-Benzyl-3-(4-methoxyphenyl)-1-oxo-2,3-dihydro-1H- benzo[4,5]thiazolo[3,2-a]pyridine-4-carbonitrile (3c)}

The ee (94\%) was measured by HPLC using a chiral stationary phase [Chiralpak AD; $n$-heptane-EtOH, 7:3, $0.5 \mathrm{~mL} / \mathrm{min} ; t_{\mathrm{R}}=18.94 \mathrm{~min}(\mathrm{mi}-$ nor), 25.78 min (major)].

Yield: $152.0 \mathrm{mg}$ (72\%); colorless solid; $\mathrm{mp} 96-98{ }^{\circ} \mathrm{C}$.

$[\alpha]_{\mathrm{D}}^{21}-500.9\left(\mathrm{c} 2.89, \mathrm{CHCl}_{3}\right)$.

IR (ATR): 3455, 3017, 2956, 2841, 2631, 2321, 2195, 2102, 2063, 2008 , 1908, 1727, 1605, 1509, 1455, 1366, 1224, 1132, 1029, 930, 826, 747. $701 \mathrm{~cm}^{-1}$.

${ }^{1} \mathrm{H}$ NMR $\left(600 \mathrm{MHz}, \mathrm{CDCl}_{3}\right): \delta=8.43(\mathrm{~d}, J=8.4 \mathrm{~Hz}, 1 \mathrm{H}), 7.41-7.22(\mathrm{~m}, 6$ $\mathrm{H}), 7.08(\mathrm{~d}, J=7.2 \mathrm{~Hz}, 2 \mathrm{H}), 6.98(\mathrm{~d}, J=8.4 \mathrm{~Hz}, 2 \mathrm{H}), 6.86(\mathrm{~d}, J=8.4 \mathrm{~Hz}$, $2 \mathrm{H}), 3.80$ (s, $3 \mathrm{H}), 3.59$ (d, $J=7.2 \mathrm{~Hz}, 1 \mathrm{H}), 3.52-3.49(\mathrm{~m}, 1 \mathrm{H}), 3.44$ $\left(\mathrm{dd}, J_{1}=14.4 \mathrm{~Hz}, J_{2}=4.8 \mathrm{~Hz}, 1 \mathrm{H}\right), 2.49\left(\mathrm{dd}, J_{1}=15.0 \mathrm{~Hz}, J_{2}=9.6 \mathrm{~Hz}, 1\right.$ $\mathrm{H})$.

${ }^{13} \mathrm{C}$ NMR $\left(150 \mathrm{MHz}, \mathrm{CDCl}_{3}\right): \delta=169.4,159.6,151.8,138.1,137.4$, 128.9 (2 C), 128.8 (2 C), 128.7 (2 C), 128.4, 127.1, 126.8, 126.0, 123.8, $121.9,118.0,117.2,114.6$ (2 C), 83.9, 55.3, 48.2, 42.4, 31.5 .

MS (ESI): $m / z=425.1315[\mathrm{M}+\mathrm{H}]^{+}$.

HRMS (ESI): $m / z[\mathrm{M}+\mathrm{Na}]^{+}$calcd for $\mathrm{C}_{26} \mathrm{H}_{20} \mathrm{~N}_{2} \mathrm{O}_{2} \mathrm{~S}: 447.1138$; found: 447.1137.

(2R,3R)-2-Benzyl-3-(3-methoxyphenyl)-1-oxo-2,3-dihydro-1Hbenzo[4,5]thiazolo[3,2-a]pyridine-4-carbonitrile (3d)

The ee (98\%) was measured by HPLC using a chiral stationary phase [Daicel IC; $n$-heptane- $i$-PrOH, 9:1, $1.0 \mathrm{~mL} / \mathrm{min} ; t_{\mathrm{R}}=11.62 \mathrm{~min}(\mathrm{mi}-$ nor), $14.60 \mathrm{~min}$ (major)].

Yield: $200.6 \mathrm{mg}$ (94\%); light yellow solid; mp $134-135^{\circ} \mathrm{C}$.

$[\alpha]_{\mathrm{D}}^{21}-491.0\left(c 1.0, \mathrm{CHCl}_{3}\right)$.

IR (ATR): 3425, 3023, 2937, 2839, 2641, 2322, 2195, 2088, 1989, 1922 , $1715,1599,1454,1312,1255,1140,1039,932,862,748,702 \mathrm{~cm}^{-1}$.

${ }^{1} \mathrm{H}$ NMR $\left(600 \mathrm{MHz}, \mathrm{CDCl}_{3}\right): \delta=8.43(\mathrm{~d}, J=7.8 \mathrm{~Hz}, 1 \mathrm{H}), 7.42-7.19(\mathrm{~m}, 7$ $\mathrm{H}), 7.08(\mathrm{~d}, J=7.8 \mathrm{~Hz}, 2 \mathrm{H}), 6.86\left(\mathrm{dd}, J_{1}=8.4 \mathrm{~Hz}, J_{2}=2.4 \mathrm{~Hz}, 1 \mathrm{H}\right), 6.67$ $(\mathrm{d}, J=7.8 \mathrm{~Hz}, 1 \mathrm{H}), 6.57(\mathrm{~s}, 1 \mathrm{H}), 3.76(\mathrm{~s}, 3 \mathrm{H}), 3.59(\mathrm{~d}, J=7.2 \mathrm{~Hz}, 1 \mathrm{H})$, $3.53-3.45(\mathrm{~m}, 2 \mathrm{H}), 2.51\left(\mathrm{dd}, J_{1}=14.4 \mathrm{~Hz}, J_{2}=9.6 \mathrm{~Hz}, 1 \mathrm{H}\right)$.

${ }^{13} \mathrm{C}$ NMR $\left(150 \mathrm{MHz}, \mathrm{CDCl}_{3}\right): \delta=169.3,160.0,152.3,138.1,137.9$, 137.4, 130.4, 128.9 (2 C), 128.7 (2 C), 127.1, 126.8, 126.0, 123.7, 121.8, $119.6,117.9,117.3,113.9,113.4,83.3,55.2,48.0,43.1,31.5$.

MS (ESI): $m / z=423.1194[\mathrm{M}-\mathrm{H}]^{-}$.

Anal. Calcd for $\mathrm{C}_{26} \mathrm{H}_{20} \mathrm{~N}_{2} \mathrm{O}_{2} \mathrm{~S}$ (424.12): C, 73.56; H, 4.75; N, 6.60. Found: C, 73.15; H, 5.00; N, 6.35 .
(2R,3R)-2-Benzyl-3-(4-chlorophenyl)-1-oxo-2,3-dihydro-1H-benzo[4,5]thiazolo[3,2-a]pyridine-4-carbonitrile (3e)

The ee (97\%) was measured by HPLC using a chiral stationary phase [Daicel IC; $n$-heptane-EtOH, 97:3, $0.7 \mathrm{~mL} / \mathrm{min} ; t_{\mathrm{R}}=13.11 \mathrm{~min}$ (minor), 17.74 min (major)]

Yield: $180.3 \mathrm{mg}(84 \%)$; colorless solid; $\mathrm{mp} 97-98^{\circ} \mathrm{C}$.

$[\alpha]_{\mathrm{D}}^{21}-596.0\left(c 1.0, \mathrm{CHCl}_{3}\right)$.

IR (ATR): 3450, 3025, 2937, 2639, 2323, 2195, 2104, 1985, 1905, 1721 $1605,1491,1458,1365,1318,1222,1134,1094,1015,934,825,747$, $701 \mathrm{~cm}^{-1}$.

${ }^{1} \mathrm{H}$ NMR $\left(600 \mathrm{MHz}, \mathrm{CDCl}_{3}\right): \delta=8.43(\mathrm{~d}, J=8.4 \mathrm{~Hz}, 1 \mathrm{H}), 7.43-7.22(\mathrm{~m}, 8$ $\mathrm{H}), 7.05(\mathrm{~d}, J=7.2 \mathrm{~Hz}, 2 \mathrm{H}), 6.98(\mathrm{~d}, J=8.4 \mathrm{~Hz}, 2 \mathrm{H}), 3.61(\mathrm{~d}, J=7.2 \mathrm{~Hz}$, $1 \mathrm{H}), 3.56-3.52(\mathrm{~m}, 1 \mathrm{H}), 3.46\left(\mathrm{dd}, J_{1}=14.4 \mathrm{~Hz}, J_{2}=4.8 \mathrm{~Hz}, 1 \mathrm{H}\right), 2.44$ (dd, $J_{1}=15.0 \mathrm{~Hz}, J_{2}=9.6 \mathrm{~Hz}, 1 \mathrm{H}$ ).

${ }^{13} \mathrm{C}$ NMR $\left(150 \mathrm{MHz}, \mathrm{CDCl}_{3}\right): \delta=169.0,152.6,137.6,137.3,134.9$, 134.4, 129.5 (2 C), 129.0 (2 C), 128.8 (2 C), 128.8 (2 C), 127.2, 127.0, $126.1,123.7,121.9,117.7,117.3,82.8,47.7,42.4,31.5$.

MS (ESI): $m / z=429.0825[\mathrm{M}+\mathrm{H}]^{+}$.

HRMS (ESI): $m / z$ [M+ H] $]^{+}$calcd for $\mathrm{C}_{25} \mathrm{H}_{17} \mathrm{ClN}_{2} \mathrm{OS}$ : 429.0823 ; found: 429.0823.

(2R,3R)-2-Benzyl-3-(3-chlorophenyl)-1-oxo-2,3-dihydro-1H-benzo[4,5]thiazolo[3,2-a]pyridine-4-carbonitrile (3f)

The ee $(98 \%)$ was measured by HPLC using a chiral stationary phase [Daicel IC; $n$-heptane-EtOH, 97:3, $1.0 \mathrm{~mL} / \mathrm{min} ; t_{\mathrm{R}}=9.42 \mathrm{~min}$ (minor), 13.16 min (major)].

Yield: $208.5 \mathrm{mg}$ (97\%); colorless solid; $\mathrm{mp} 85-86{ }^{\circ} \mathrm{C}$.

$[\alpha]_{D}^{21}-506.3\left(c\right.$ 1.0, $\left.\mathrm{CHCl}_{3}\right)$

IR (ATR): 3441, 3025, 2929, 2640, 2322, 2195, 2101, 1979, 1718, 1601, $1458,1362,1315,1221,1135,1081,870,747,698 \mathrm{~cm}^{-1}$.

${ }^{1} \mathrm{H}$ NMR $\left(600 \mathrm{MHz}, \mathrm{CDCl}_{3}\right): \delta=8.43(\mathrm{~d}, J=7.8 \mathrm{~Hz}, 1 \mathrm{H}), 7.43-7.22(\mathrm{~m}, 8$ H), $7.06(\mathrm{~d}, J=7.2 \mathrm{~Hz}, 2 \mathrm{H}), 7.00-6.99(\mathrm{~m}, 1 \mathrm{H}), 6.95(\mathrm{~d}, J=7.2 \mathrm{~Hz}, 1 \mathrm{H})$, $3.61(\mathrm{~d}, J=7.2 \mathrm{~Hz}, 1 \mathrm{H}), 3.57-3.53(\mathrm{~m}, 1 \mathrm{H}), 3.47\left(\mathrm{dd}, J_{1}=15.0 \mathrm{~Hz}\right.$, $\left.J_{2}=4.8 \mathrm{~Hz}, 1 \mathrm{H}\right), 2.45\left(\mathrm{dd}, J_{1}=14.4 \mathrm{~Hz}, J_{2}=9.6 \mathrm{~Hz}, 1 \mathrm{H}\right.$ ).

${ }^{13} \mathrm{C}$ NMR $\left(150 \mathrm{MHz}, \mathrm{CDCl}_{3}\right): \delta=168.9,152.9,138.4,137.6,137.3$, 134.9, 130.6, 129.1, 129.1, 128.8 (2 C), 128.8 (2 C), 128.7, 128.1, 127.2, $127.0,126.1,125.6,121.9,117.3,82.5,47.7,42.7,31.5$

MS (ESI): $m / z=429.0825[\mathrm{M}+\mathrm{H}]^{+}$.

Anal. Calcd for $\mathrm{C}_{25} \mathrm{H}_{17} \mathrm{ClN}_{2} \mathrm{OS}$ (428.08): C, 70.00; H, 3.99; N, 6.53. Found: C, 69.85; H, 3.82; N, 6.65.

(2R,3R)-2-Benzyl-3-(4-fluorophenyl)-1-oxo-2,3-dihydro-1H-benzo[4,5]thiazolo[3,2-a]pyridine-4-carbonitrile (3g)

The ee $(97 \%)$ was measured by HPLC using a chiral stationary phase [Daicel IA; $n$-heptane-EtOH, 9:1, $1.0 \mathrm{~mL} / \mathrm{min} ; t_{\mathrm{R}}=11.42 \mathrm{~min}$ (minor), $17.83 \mathrm{~min}$ (major)]

Yield: $201.3 \mathrm{mg}$ (97\%); light yellow solid; $\mathrm{mp} 64-66{ }^{\circ} \mathrm{C}$.

$[\alpha]_{\mathrm{D}}^{21}-486.3\left(\right.$ c $\left.1.0, \mathrm{CHCl}_{3}\right)$

IR (ATR): 3448, 3025, 2939, 2651, 2323, 2196, 2106, 1894, 1720, 1603 , $1506,1457,1363,1315,1223,1138,1026,911,831,743 \mathrm{~cm}^{-1}$.

${ }^{1} \mathrm{H} \mathrm{NMR}\left(600 \mathrm{MHz}, \mathrm{CDCl}_{3}\right): \delta=8.43\left(\mathrm{dd}, J_{1}=7.8 \mathrm{~Hz}, J_{2}=0.6 \mathrm{~Hz}, 1 \mathrm{H}\right)$, 7.42-7.22 (m, $7 \mathrm{H}), 7.05-6.96(\mathrm{~m}, 5 \mathrm{H}), 3.63$ (d, J= 7.2 Hz, $1 \mathrm{H}), 3.56-$ $3.52(\mathrm{~m}, 1 \mathrm{H}), 3.45\left(\mathrm{dd}, J_{1}=15.0 \mathrm{~Hz}, J_{2}=4.8 \mathrm{~Hz}, 1 \mathrm{H}\right), 2.45\left(\mathrm{dd}, J_{1}=14.4\right.$ $\left.\mathrm{Hz}, J_{2}=9.6 \mathrm{~Hz}, 1 \mathrm{H}\right)$.

${ }^{13} \mathrm{C}$ NMR $\left(100 \mathrm{MHz}, \mathrm{CDCl}_{3}\right): \delta=169.1,162.6\left(\mathrm{~d}, J_{\mathrm{F}-\mathrm{C}}=246 \mathrm{~Hz}\right), 152.4$, 137.7, 137.3, 132.3 (d, $\left.J_{F-C}=4 \mathrm{~Hz}\right), 129.4\left(\mathrm{~d}, J_{F-C}=8 \mathrm{~Hz}, 2 \mathrm{C}\right), 128.8(2$ 
C), 128.7 (2 C), 127.2, 126.9, 126.1, 123.7, 121.9, 117.7, 117.3, 116.2 $\left(\mathrm{d}, J_{F-C}=22 \mathrm{~Hz}, 2 \mathrm{C}\right), 83.2,47.9,42.3,31.5$.

MS (ESI): $m / z=413.1121[\mathrm{M}+\mathrm{H}]^{+}$.

HRMS (ESI): $m / z[\mathrm{M}+\mathrm{H}]^{+}$calcd for $\mathrm{C}_{25} \mathrm{H}_{17} \mathrm{FN}_{2} \mathrm{OS}$ : 413.1118; found: 413.1119.

\section{(2R,3S)-2-Benzyl-3-(furan-2-yl)-1-oxo-2,3-dihydro-1H-ben-} zo[4,5]thiazolo[3,2-a]pyridine-4-carbonitrile (3h)

The ee $(92 \%)$ was measured by HPLC using a chiral stationary phase [Daicel IC; $n$-heptane-EtOH, 97:3, $1.0 \mathrm{~mL} / \mathrm{min} ; t_{\mathrm{R}}=11.21 \mathrm{~min}$ (minor), $13.72 \mathrm{~min}$ (major)].

Yield: $129.8 \mathrm{mg}(68 \%)$; colorless solid; $\mathrm{mp} 76-78{ }^{\circ} \mathrm{C}$.

$[\alpha]_{\mathrm{D}}^{21}-444.6\left(\right.$ c $\left.1.0, \mathrm{CHCl}_{3}\right)$.

IR (ATR): 3781, 3118, 3065, 3027, 2933, 2719, 2345, 2197, 2040, 1990, 1963, 1790, 1719, 1606, 1579, 1497, 1458, 1361, 1318, 1256, 1227 , $1135,1066,1012,922,865,814,789,743,702 \mathrm{~cm}^{-1}$.

${ }^{1} \mathrm{H}$ NMR $\left(600 \mathrm{MHz}, \mathrm{CDCl}_{3}\right): \delta=8.45(\mathrm{~d}, J=8.4 \mathrm{~Hz}, 1 \mathrm{H}), 7.40-7.22(\mathrm{~m}, 7$ $\mathrm{H}), 7.13(\mathrm{~d}, J=7.8 \mathrm{~Hz}, 2 \mathrm{H}), 6.35-6.34(\mathrm{~m}, 1 \mathrm{H}), 6.19(\mathrm{~d}, J=3.0 \mathrm{~Hz}, 1 \mathrm{H})$, $3.67\left(\mathrm{~d}, J=6.6 \mathrm{~Hz}, 1 \mathrm{H}\right.$ ), $3.54\left(\mathrm{dd}, J_{1}=14.4 \mathrm{~Hz}, J_{2}=4.2 \mathrm{~Hz}, 1 \mathrm{H}\right), 3.36-$ $3.32(\mathrm{~m}, 1 \mathrm{H}), 2.41\left(\mathrm{dd}, J_{1}=14.4 \mathrm{~Hz}, J_{2}=10.8 \mathrm{~Hz}, 1 \mathrm{H}\right)$.

${ }^{13} \mathrm{C}$ NMR $\left(150 \mathrm{MHz}, \mathrm{CDCl}_{3}\right): \delta=168.8,153.7,149.7,143.1,138.0$, 137.7, 129.0 (2 C), 128.8 (2 C), 127.1, 126.9, 125.8, 123.7, 121.8, 117.8, $117.4,110.4,109.0,79.9,47.4,36.2,31.7$

MS (ESI): $m / z=383.0845[\mathrm{M}-\mathrm{H}]^{-}$.

HRMS (ESI): $m / z$ [M+H] calcd for $\mathrm{C}_{23} \mathrm{H}_{16} \mathrm{~N}_{2} \mathrm{O}_{2} \mathrm{~S}: 385.1005$; found: 385.1005 .

(2R,3R)-2-Benzyl-8-chloro-1-oxo-3-phenyl-2,3-dihydro-1H-benzo[4,5]thiazolo[3,2-a]pyridine-4-carbonitrile (3i)

The ee $(98 \%)$ was measured by HPLC using a chiral stationary phase [Daicel IC; $n$-heptane-EtOH, 97:3, $0.5 \mathrm{~mL} / \mathrm{min} ; t_{\mathrm{R}}=19.67 \mathrm{~min}$ (minor), 27.51 min (major)].

Yield: $208.3 \mathrm{mg}$ (97\%); colorless solid; mp $240-242{ }^{\circ} \mathrm{C}$.

$[\alpha]_{\mathrm{D}}^{21}-597.3\left(c 1.0, \mathrm{CHCl}_{3}\right)$.

IR (ATR): 3413, 3124, 3084, 3029, 2920, 2877, 2318, 2190, 2037, 1993 , 1955, 1825, 1714, 1602, 1573, 1493, 1457, 1418, 1368, 1297, 1267 , $1221,1135,1080,1052,996,939,896,865,805,752,698,663 \mathrm{~cm}^{-1}$.

${ }^{1} \mathrm{H}$ NMR $\left(400 \mathrm{MHz}, \mathrm{CDCl}_{3}\right): \delta=8.47(\mathrm{~d}, J=2.0 \mathrm{~Hz}, 1 \mathrm{H}), 7.34-7.20(\mathrm{~m}, 8$ H), 7.05-7.02 (m, $4 \mathrm{H}), 3.63(\mathrm{~d}, J=6.8 \mathrm{~Hz}, 1 \mathrm{H}), 3.54-3.49(\mathrm{~m}, 1 \mathrm{H})$, $3.41\left(\mathrm{dd}, J_{1}=14.4 \mathrm{~Hz}, J_{2}=4.4 \mathrm{~Hz}, 1 \mathrm{H}\right), 2.48\left(\mathrm{dd}, J_{1}=14.8 \mathrm{~Hz}, J_{2}=9.6\right.$ $\mathrm{Hz}, 1 \mathrm{H})$.

${ }^{13} \mathrm{C}$ NMR $\left(100 \mathrm{MHz}, \mathrm{CDCl}_{3}\right): \delta=169.2,152.0,138.2,137.9,136.2$, 133.2, 129.3 (2 C), 128.8 (2 C), 128.7 (2 C), 128.6, 127.6 (2 C), 126.9, $126.0,122.3,122.2,117.7,117.5,84.3,48.0,43.1,31.5$.

MS (ESI): $m / z=427.0682[\mathrm{M}-\mathrm{H}]^{-}$.

HRMS (ESI): $m / z$ [M $+\mathrm{H}]^{+}$calcd for $\mathrm{C}_{25} \mathrm{H}_{17} \mathrm{ClN}_{2} \mathrm{OS}$ : 429.0823; found: 429.0817.

(2R,3R)-2-Benzyl-1-oxo-3-phenyl-2,3-dihydro-1H-benzo[4,5]oxazolo[3,2-a]pyridine-4-carbonitrile (3j)

The ee (95\%) was measured by HPLC using a chiral stationary phase [Daicel IC; $n$-heptane-EtOH, 97:3, $0.7 \mathrm{~mL} / \mathrm{min} ; t_{\mathrm{R}}=19.07 \mathrm{~min}$ (minor), 26.35 min (major)].

Yield: $183.8 \mathrm{mg}$ (97\%); colorless solid; $\mathrm{mp} 138-140{ }^{\circ} \mathrm{C}$.

$[\alpha]_{\mathrm{D}}^{21}-445.9\left(c 1.0, \mathrm{CHCl}_{3}\right)$
IR (ATR): 3448, 3028, 2942, 2320, 2204, 2103, 1967, 1893, 1692, 1621, 1471, 1368, 1263, 1225, 1192, 1128, 1089, 1006, 923, 843, 745, 697 $\mathrm{cm}^{-1}$.

${ }^{1} \mathrm{H}$ NMR $\left(600 \mathrm{MHz}, \mathrm{CDCl}_{3}\right): \delta=8.01-7.98(\mathrm{~m}, 1 \mathrm{H}), 7.34-7.24(\mathrm{~m}, 9 \mathrm{H})$, 7.07-7.06 (m, $2 \mathrm{H}), 7.03$ (d, $J=7.2 \mathrm{~Hz}, 2 \mathrm{H}), 3.68-3.67(\mathrm{~m}, 1 \mathrm{H}), 3.51-$ 3.46 (m, $2 \mathrm{H}), 2.50-2.46$ ( $\mathrm{m}, 1 \mathrm{H})$.

${ }^{13} \mathrm{C}$ NMR $\left(150 \mathrm{MHz}, \mathrm{CDCl}_{3}\right): \delta=167.6,156.9,146.9,137.9,137.2$, 129.3 (2 C), 128.8 (2 C), 128.7 (2 C), 128.4, 127.4 (2 C), 127.1, 126.9, $125.8,125.0,116.0,114.4,110.4,66.0,47.9,41.3,31.2$.

MS (ESI): $m / z=401.1860[\mathrm{M}+\mathrm{Na}]^{+}$.

Anal. Calcd for $\mathrm{C}_{25} \mathrm{H}_{18} \mathrm{~N}_{2} \mathrm{O}_{2}$ (378.14): C, 79.35; H, 4.79; N, 7.40. Found: C, 79.06; H, 4.94; N, 7.29.

(2R,3R)-2-Butyl-1-oxo-3-phenyl-2,3-dihydro-1H-benzo[4,5]thiazolo[3,2-a]pyridine-4-carbonitrile (3k)

The ee $(98 \%)$ was measured by HPLC using a chiral stationary phase [Daicel IC; $n$-heptane-EtOH, 97:3, $0.5 \mathrm{~mL} / \mathrm{min} ; t_{\mathrm{R}}=15.59 \mathrm{~min}$ (minor), 19.09 min (major)]

Yield: $128.1 \mathrm{mg}$ (71\%); colorless solid; $\mathrm{mp} 100-102{ }^{\circ} \mathrm{C}$.

$[\alpha]_{D}^{21}-511.7$ ( c 0.96, $\left.\mathrm{CHCl}_{3}\right)$.

IR (ATR): 3423, 3029, 2948, 2865, 2196, 1947, 1717, 1606, 1457, 1310, $1226,1135,1060,1028,908,846,740 \mathrm{~cm}^{-1}$.

${ }^{1} \mathrm{H}$ NMR $\left(600 \mathrm{MHz}, \mathrm{CDCl}_{3}\right): \delta=8.40(\mathrm{~d}, J=7.8 \mathrm{~Hz}, 1 \mathrm{H}), 7.40-7.23(\mathrm{~m}, 6$ $\mathrm{H}), 7.12\left(\mathrm{dd}, J_{1}=7.8 \mathrm{~Hz}, J_{2}=2.4 \mathrm{~Hz}, 2 \mathrm{H}\right), 3.84(\mathrm{~d}, J=7.2 \mathrm{~Hz}, 1 \mathrm{H}), 3.09$ $\left(\mathrm{dd}, J_{1}=13.2 \mathrm{~Hz}, J_{2}=7.2 \mathrm{~Hz}, 1 \mathrm{H}\right), 1.94-1.88(\mathrm{~m}, 1 \mathrm{H}), 1.42-1.21(\mathrm{~m}, 5$ $\mathrm{H}), 0.88(\mathrm{t}, J=7.2 \mathrm{~Hz}, 3 \mathrm{H})$.

${ }^{13} \mathrm{C}$ NMR $\left(150 \mathrm{MHz}, \mathrm{CDCl}_{3}\right): \delta=169.8,152.5,137.6,136.5,129.2(2 \mathrm{C})$, 128.3, 127.4 (2 C), 127.1, 125.9, 123.8, 121.8, 118.1, 117.2, 83.0, 46.5, 43.9, 29.5, 25.4, 22.5, 13.9 .

MS (ESI): $m / z=359.1202[\mathrm{M}-\mathrm{H}]^{-}$.

HRMS (ESI): $m / z[\mathrm{M}+\mathrm{Na}]^{+}$calcd for $\mathrm{C}_{22} \mathrm{H}_{20} \mathrm{~N}_{2} \mathrm{OS}$ : 383.1196; found: 383.1187.

(2R,3R)-2-Hexyl-1-oxo-3-phenyl-2,3-dihydro-1H-benzo[4,5]thiazolo[3,2-a]pyridine-4-carbonitrile (31)

The ee $(96 \%)$ was measured by HPLC using a chiral stationary phase [Daicel IC; $n$-heptane-EtOH, 97:3, $0.7 \mathrm{~mL} / \mathrm{min} ; t_{\mathrm{R}}=12.62 \mathrm{~min}$ (minor), $14.81 \mathrm{~min}$ (major)].

Yield: $85.5 \mathrm{mg}$ (44\%); colorless solid; $\mathrm{mp} 123-125^{\circ} \mathrm{C}$.

$[\alpha]_{D}^{21}-448.6\left(c 1.0, \mathrm{CHCl}_{3}\right)$.

IR (ATR): 3427, 3029, 2927, 2858, 2645, 2321, 2196, 2104, 1993, 1804 , $1717,1607,1457,1309,1225,1135,1062,1028,908,850,744 \mathrm{~cm}^{-1}$.

${ }^{1} \mathrm{H}$ NMR $\left(600 \mathrm{MHz}, \mathrm{CDCl}_{3}\right): \delta=8.40(\mathrm{~d}, J=8.4 \mathrm{~Hz}, 1 \mathrm{H}), 7.38(\mathrm{~d}, J=7.8$ $\mathrm{Hz}, 1 \mathrm{H}), 7.32-7.23(\mathrm{~m}, 5 \mathrm{H}), 7.12\left(\mathrm{dd}, J_{1}=7.8 \mathrm{~Hz}, J_{2}=2.4 \mathrm{~Hz}, 2 \mathrm{H}\right), 3.84$ (d, $J=7.2 \mathrm{~Hz}, 1 \mathrm{H}$ ), 3.09 (dd, $J_{1}=13.2 \mathrm{~Hz}, J_{2}=7.2 \mathrm{~Hz}, 1 \mathrm{H}$ ), $1.93-1.87$ (m, $1 \mathrm{H}), 1.43-1.38(\mathrm{~m}, 2 \mathrm{H}), 1.31-1.20(\mathrm{~m}, 7 \mathrm{H}), 0.86(\mathrm{t}, J=7.2 \mathrm{~Hz}, 3$ $\mathrm{H})$.

${ }^{13} \mathrm{C}$ NMR $\left(150 \mathrm{MHz}, \mathrm{CDCl}_{3}\right): \delta=169.8,152.5,137.6,136.5,129.2(2 \mathrm{C})$, $128.3,127.4$ (2 C), 127.1, 125.9, 123.7, 121.8, 118.1, 117.2, 83.0, 46.5, 43.9, 31.5, 29.1, 27.3, 25.7, 22.5, 14.0.

MS (ESI): $m / z=387.1536[\mathrm{M}-\mathrm{H}]^{-}$.

HRMS (ESI): $m / z[\mathrm{M}+\mathrm{Na}]^{+}$calcd for $\mathrm{C}_{24} \mathrm{H}_{24} \mathrm{~N}_{2} \mathrm{OS}$ : 411.1504; found: 411.1502 . 


\section{Acknowledgment}

We thank the European Research Council (ERC Advanced Grant 320493 'DOMINOCAT') for financial support. X.S. and Q.N. are grateful to the China Scholarship Council for a fellowship.

\section{Supporting Information}

Supporting information for this article is available online at http://dx.doi.org/10.1055/s-0034-1379369. Included are copies of the ${ }^{1} \mathrm{H}$ and ${ }^{13} \mathrm{C}$ NMR spectra of products 3a-l, HPLC measurements of products $\mathbf{3 a - 1}$, and the NOESY spectrum of $\mathbf{3 d}$.

\section{References}

(1) (a) Girling, P. R.; Kiyoi, T.; Whiting, A. Org. Biomol. Chem. 2011, 9, 3105. (b) Masson, G.; Lalli, C.; Benohoud, M.; Dagousset, G. Chem. Soc. Rev. 2013, 42, 902.

(2) Serckx-Poncin, B.; Hesbain-Frisque, A.-M.; Ghosez, L. Tetrahedron Lett. 1982, 23, 3261.

(3) (a) Danishefsky, S.; Kerwin, J. F. Jr. J. Org. Chem. 1982, 47, 3183. (b) Kerwin, J. F. Jr.; Danishefsky, S. Tetrahedron Lett. 1982, 23, 3739.

(4) For selected examples, see: (a) Shang, D.; Xin, J.; Liu, Y.; Zhou, X.; Liu, X.; Feng, X. J. Org. Chem. 2008, 73, 630. (b) Sridharan, V.; Perumal, P. T.; Avendaño, C.; Menéndez, J. C. Org. Biomol. Chem. 2007, 5, 1351. (c) Xie, M.; Chen, X.; Zhu, Y.; Gao, B.; Lin, L.; Liu, X.; Feng, X. Angew. Chem. Int. Ed. 2010, 49, 3799. (d) Wakabayashi, R.; Kurahashi, T.; Matsubara, S. Org. Lett. 2012, 14, 4794. (e) Deng, Y.; Liu, L.; Sarkisian, R. G.; Wheeler, K.; Wang, H.; Xu, Z. Angew. Chem. Int. Ed. 2013, 52, 3663. (f) Eschenbrenner-Lux, V.; Küchler, P.; Ziegler, S.; Kumar, K.; Waldmann, H. Angew. Chem. Int. Ed. 2014, 53, 2134.

(5) (a) Itoh, T.; Yokoya, M.; Miyauchi, K.; Nagata, K.; Ohsawa, A. Org. Lett. 2003, 5, 4301. (b) Itoh, T.; Yokoya, M.; Miyauchi, K.; Nagata, K.; Ohsawa, A. Org. Lett. 2006, 8, 1533. (c) Han, B.; Li, J.-L.; Ma, C.; Zhang, S.-J.; Chen, Y.-C. Angew. Chem. Int. Ed. 2008, 47, 9971. (d) Han, B.; He, Z.-Q.; Li, J.-L.; Li, R.; Jiang, K.; Liu, T.-Y.; Chen, Y.C. Angew. Chem. Int. Ed. 2009, 48, 5474. (e) Li, J.-L.; Zhou, S.-L.; Han, B.; Wu, L.; Chen, Y.-C. Chem. Commun. 2010, 46, 2665. (f) Zhou, S.-L.; Li, J.-L.; Dong, L.; Chen, Y.-C. Org. Lett. 2011, 13, 5874. (g) Gu, J.; Ma, C.; Li, Q.-Z.; Du, W.; Chen, Y.-C. Org. Lett. 2014, 16, 3986.

(6) Lalonde, M. P.; McGowan, M. A.; Rajapaksa, N. S.; Jacobsen, E. N. J. Am. Chem. Soc. 2013, 135, 1891.

(7) (a) Itoh, J.; Fuchibe, K.; Akiyama, T. Angew. Chem. Int. Ed. 2006, 45, 4796. (b) Akiyama, T.; Morita, H.; Fuchibe, K. J. Am. Chem. Soc. 2006, 128, 13070. (c) He, L.; Bekkaye, M.; Retailleau, P.; Masson, G. Org. Lett. 2012, 14, 3158. (d) He, L.; Laurent, G.; Retailleau, P.; Folléas, B.; Brayer, J.-L.; Masson, G. Angew. Chem. Int. Ed. 2013, 52, 11088. (e) Brioche, J.; Courant, T.; Alcaraz, L.; Stocks, M.; Furber, M.; Zhu, J.; Masson, G. Adv. Synth. Catal. 2014, 356, 1719.

(8) For selected reviews, see: (a) Enders, D.; Balensiefer, T. Acc. Chem. Res. 2004, 37, 534. (b) Enders, D.; Niemeier, O.; Henseler, A. Chem. Rev. 2007, 107, 5606. (c) Biju, A. T.; Kuhl, N.; Glorius, F. Acc. Chem. Res. 2011, 44, 1182. (d) Izquierdo, J.; Hutson, G. E.; Cohen, D. T.; Scheidt, K. A. Angew. Chem. Int. Ed. 2012, 51, 11686. (e) Grossmann, A.; Enders, D. Angew. Chem. Int. Ed. 2012, 51, 314. (f) Bugaut, X.; Glorius, F. Chem. Soc. Rev. 2012, 41, 3511. (g) Ryan, S. J.; Candish, L.; Lupton, D. W. Chem. Soc. Rev. 2013,
42, 4906. (h) De Sarkar, S.; Biswas, A.; Samanta, R. C.; Studer, A. Chem. Eur. J. 2013, 19, 4664. (i) Mahatthananchai, J.; Bode, J. W. Acc. Chem. Res. 2014, 47, 696. (j) Hopkinson, M. N.; Richter, C.; Schedler, M.; Glorius, F. Nature 2014, 510, 485. (k) Abbasov, M. E.; Romo, D. Nat. Prod. Rep. 2014, 31, 1318.

(9) (a) Burstein, C.; Glorius, F. Angew. Chem. Int. Ed. 2004, 43, 6205. (b) Sohn, S. S.; Rosen, E. L.; Bode, J. W. J. Am. Chem. Soc. 2004, 126, 14370. For a review, see: (c) Nair, V.; Menon, R. S.; Biju, A. T.; Sinu, C. R.; Paul, R. R.; Jose, A.; Sreekumar, V. Chem. Soc. Rev. 2011, 40, 5336.

(10) (a) Reynolds, N. T.; Rovis, T. J. Am. Chem. Soc. 2005, 127, 16406. (b) Douglas, J.; Churchill, G.; Smith, A. D. Synthesis 2012, 44, 2295. (c) Vora, H. U.; Wheeler, P.; Rovis, T. Adv. Synth. Catal. 2012, 354, 1617.

(11) (a) Zhang, Y.-R.; He, L.; Wu, X.; Shao, P.-L.; Ye, S. Org. Lett. 2008, 10, 277. (b) Duguet, N.; Campbell, C. D.; Slawin, A. M. Z.; Smith, A. D. Org. Biomol. Chem. 2008, 6, 1108. (c) He, L.; Lv, H.; Zhang, Y.-R.; Ye, S. J. Org. Chem. 2008, 73, 8101. (d) Wang, X.-N.; Shao, P.-L.; Lv, H.; Ye, S. Org. Lett. 2009, 11, 4029. (e) Huang, X.-L.; Chen, X.-Y.; Ye, S. J. Org. Chem. 2009, 74, 7585. (f) Wang, X.-N.; Zhang, Y.-Y.; Ye, S. Adv. Synth. Catal. 2010, 352, 1892. (g) Wang, T.; Huang, X.-L.; Ye, S. Org. Biomol. Chem. 2010, 8, 5007. (h) Jian, T.-Y.; He, L.; Tang, C.; Ye, S. Angew. Chem. Int. Ed. 2011, 50, 9104. (i) Zhang, H.-M.; Gao, Z.-H.; Ye, S. Org. Lett. 2014, 16, 3079.

(12) (a) Shao, P.-L.; Chen, X.-Y.; Ye, S. Angew. Chem. Int. Ed. 2010, 49, 8412. (b) Li, L.; Du, D.; Ren, J.; Wang, Z. Eur. J. Org. Chem. 2011, 614. (c) Ni, Q.; Zhang, H.; Grossmann, A.; Loh, C. C. J.; Merkens, C.; Enders, D. Angew. Chem. Int. Ed. 2013, 52, 13562.

(13) (a) He, M.; Uc, G. J.; Bode, J. W. J. Am. Chem. Soc. 2006, 128, 15088. (b) Zhang, Y.-R.; Lv, H.; Zhou, D.; Ye, S. Chem. Eur. J. 2008, 14, 8473. (c) Huang, X.-L.; He, L.; Shao, P.-L.; Ye, S. Angew. Chem. Int. Ed. 2009, 48, 192. (d) Kaeobamrung, J.; Kozlowski, M. C.; Bode, J. W. Proc. Natl. Acad. Sci. U.S.A. 2010, 107, 20661. (e) Lv, H.; Chen, X.-Y.; Sun, L.-H.; Ye, S. J. Org. Chem. 2010, 75, 6973. (f) Fang, X.; Chen, X.; Chi, Y. R. Org. Lett. 2011, 13, 4708. (g) Lv, H.; Mo, J.; Fang, X.; Chi, Y. R. Org. Lett. 2011, 13, 5366. (h) Yang, L.; Wang, F.; Chua, P. J.; Lv, Y.; Zhong, L.-J.; Zhong, G. Org. Lett. 2012, 14, 2894. (i) Allen, S. E.; Mahatthananchai, J.; Bode, J. W.; Kozlowski, M. C. J. Am. Chem. Soc. 2012, 134, 12098. (j) Yang, L.; Wang, F.; Lee, R.; Lv, Y.; Huang, K.-W.; Zhong, G. Org. Lett. 2014, $16,3872$.

(14) He, M.; Struble, J. R.; Bode, J. W. J. Am. Chem. Soc. 2006, 128, 8418.

(15) Jian, T.-Y.; Shao, P.-L.; Ye, S. Chem. Commun. 2011, 47, 2381.

(16) Jian, T.-Y.; Sun, L.-H.; Ye, S. Chem. Commun. 2012, 48, 10907.

(17) Zhao, X.; Ruhl, K. E.; Rovis, T. Angew. Chem. Int. Ed. 2012, 51, 12330.

(18) (a) Hao, L.; Du, Y.; Lv, H.; Chen, X.; Jiang, H.; Shao, Y.; Chi, Y. R. Org. Lett. 2012, 14, 2154. (b) Hao, L.; Chuen, C. W.; Ganguly, R.; Chi, Y. R. Synlett 2013, 24, 1197.

(19) Haddach, M.; Schwaebe, M. K.; Michaux, J.; Nagasawa, J.; O’Brien, S. E.; Whitten, J. P.; Pierre, F.; Kerdoncuff, P.; Darjania, L.; Stansfield, R.; Drygin, D.; Anderes, K.; Proffitt, C.; Bliesath, J.; Siddiqui-Jain, A.; Omori, M.; Huser, N.; Rice, W. G.; Ryckman, D. M. ACS Med. Chem. Lett. 2012, 3, 602.

(20) Dinakaran, M.; Senthilkumar, P.; Yogeeswari, P.; China, A.; Nagaraja, V.; Sriram, D. Bioorg. Med. Chem. 2008, 16, 3408.

(21) CCDC 1016815 contains the supplementary crystallographic data for compound 3d reported in this paper. These data can be obtained free of charge from The Cambridge Crystallographic Data Centre via www.ccdc.cam.ac.uk/data_request/cif.

(22) Leach, A. G.; Houk, K. N. J. Org. Chem. 2001, 66, 5192. 
Synthesis

X. Song et al.

(23) (a) Thomson, J. E.; Campbell, C. D.; Concellon, C.; Duguet, N.; Rix, K.; Slawin, A. M. Z.; Smith, A. D. J. Org. Chem. 2008, 73, 2784. (b) Vora, H. U.; Lathrop, S. P.; Reynolds, N. T.; Kerr, M. S.; Read de Alaniz, J.; Rovis, T. Org. Synth. 2010, 87, 350. (c) Struble, J. R.; Bode, J. W. Org. Synth. 2010, 87, 362. (d) Ling, K. B.; Smith, A. D. Chem. Commun. 2011, 47, 373. (e) Campbell, C. D.; Concellón, C.; Smith, A. D. Tetrahedron: Asymmetry 2011, 22, 797.
(24) (a) Saito, K.; Kambe, S.; Nakano, Y. Synthesis 1983, 210. (b) Fathy, N. M.; Abdel Motti, F. M.; Elgemeie, G. E. H. Arch. Pharm. (Weinheim, Ger.) 1988, 321, 509.

(25) (a) Borg, T.; Danielsson, J.; Somfai, P. Chem. Commun. 2010, 46, 1281. (b) Halland, N.; Braunton, A.; Bachmann, S.; Marigo, M.; Jørgensen, K. A. J. Am. Chem. Soc. 2004, 126, 4790. 